\title{
Evaluation of Experimental Conditions for Sample Derivatization to Identify the Metabolomic Profile of Urine by GC-MS
}

\author{
Felipe M. Aggio*, Gustavo Henrique B. Duarte, Ronei J. Poppi, Marcos N. Eberlin, Ana Valéria C. Simionato.
}

\begin{abstract}
Analyses of biological samples by GC-MS requires sample derivatization so that non-volatile or thermally unstable species may be detected. In metabolomic investigation, optimization of derivatization conditions aims to obtain the maximum number of molecular features. For this purpose, urine samples were prepared varying the reagents volumes and time of reaction, and the results were evaluated by response surfaces, indicating the most approppriate conditions.
\end{abstract}

Key words: Metabolomics profile, GC-MS, Factorial planning.

\section{Introduction}

Metabolites are biomolecules of low molecular mass with various organic function, which are formed as intermediates of biochemical reactions. These molecules are concentrated and excreted by urine. The metabolites profile and concentrations may be related to the physiological state of an organism. ${ }^{1}$ Gas chromatography coupled to mass spectrometry (GC-MS) may be used in metabolomics studies due to high resolution, sensitivity and reliable identification of metabolites. However, metabolites derivatization is required in order to obtain volatile and thermally stable species. $^{2}$ In this work optimization of sample preparation was performed by variation of three factors in two levels $([+],[-])$, thus executing a factorial planning $\left(2^{3}\right)$, that allows analyzing how the experimental variables interact and which condition generates the largest number of molecular features. ${ }^{3}$

\section{Results and Discussion}

A pool of 51 urine samples from healthy individuals were prepared and stored at $-80{ }^{\circ} \mathrm{C}$. Sample preparation was based on Barbas et al. protocol ${ }^{4}$, and the experimental variables were: 1) methoximation agent volume; 2) time of methoximation reaction and 3 ) silylating agent volume. Urea was decomposed and proteins were precipitated prior to sample derivatization. Sample supernatant was dried and reacted with $30 \mu \mathrm{L}$ (level [-]) or $40 \mu \mathrm{L}$ (level [+]) of O-methoxyamine hydrochloride (methoximation). Reaction was left for $14 \mathrm{~h}$ (level [-]) or $16 \mathrm{~h}$ (level [+]). Different volumes of silylating agent (MSTFA with $1 \%$ TMCS) was added: $30 \mu \mathrm{L}$ (level [-]) or $40 \mu \mathrm{L}$ (level [+]), and reacted for $1 \mathrm{~h}$. Samples were resuspended in $40 \mu \mathrm{L}$ of $\mathrm{n}$ heptane and analyzed by GC-MS.

The GC-MS analysis conditions was based on Fiehn et al. methodology ${ }^{2}$. Briefly: injection volume: $1 \mu \mathrm{L}$; splitless mode; initial temp.: $60^{\circ} \mathrm{C}$; temp. gradient: $10^{\circ} \mathrm{C} \mathrm{min}^{-1}$; final temp.: $325^{\circ} \mathrm{C}$; MSD transfer line temp.: $290^{\circ} \mathrm{C}$; scan: $[\mathrm{m} / \mathrm{z}$ 50.0 - 600.00]. The analysis was performed in triplicate, resulting in 24 samples, plus five quality controls and two blanks. Metabolomic data treatment is underway using Agilent MassHunter Qualitative Analysis and Agilent Unkowns Analysis software. Chemometrics treatment was performed with MatLab (The MathWorks, Natick, MA) to obtain the matrix of results (number of ion peaks). A linear statistic model was constructed respecting the equation below and was represented by a plane that relates all the variables simultaneously with the response of analysis ${ }^{3}$ (number of molecular features).

$$
\hat{y}=b_{0}+b_{1} x_{1}+b_{2} x_{2}+b_{3} x_{3}+b_{12} x_{1} x_{2}+b_{13} x_{1} x_{3}+b_{23} x_{2} x_{3}+b_{123} x_{1} x_{2} x_{3}
$$

Where $b_{n}$ are the coefficients of the equation and $x_{m}$ are the effects of each variable. The response surfaces were plotted (Image 1).

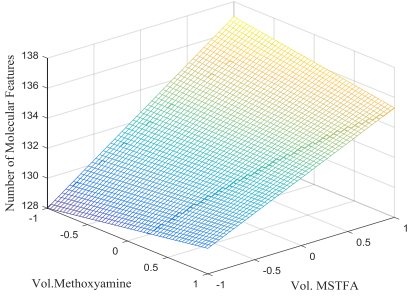

(a)

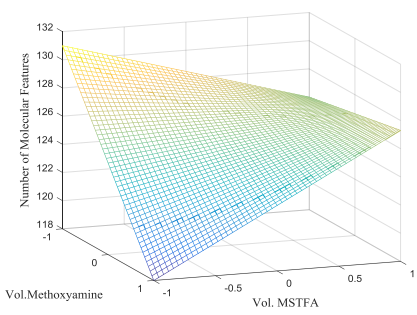

(b)
Image 1. Response surfaces based on variable 2 - time of methoximation reaction: (a) level [-]; (b) level [+].

\section{Conclusions}

Response surfaces have shown that the best conditions of sample preparation were achieved with $30 \mu \mathrm{L}$ of the methoximation agent, reacting for $14 \mathrm{~h}$ and with $40 \mu \mathrm{L}$ of silylating agent. Further optimization will be still performed to evaluate new levels of the presented factors as well as other experimental conditions.

\section{Acknowledgement}

\section{To FAPESP (proc. ํㅜ 2016/07014-5) and CNPq.}

\footnotetext{
${ }^{1}$ VILLAS-BÔAS, S. G. et al. Metabolome Analysis: An Introduction. $1^{\mathrm{a}}$ ed. John Wiley \& Sons, Inc. Hoboken, NJ. 2007

${ }^{2}$ Agilent Technologies, Inc. Agilent G1676AA Fiehn GC/MS Metabolomics RTL Library. Santa Clara, CA. 2013.

${ }^{3}$ BARROS NETO, B. et al. Como fazer experimentos: pesquisa $e$ desenvolvimento na ciência e na indústria. $3^{\text {a }}$ ed. Editora da Unicamp. Campinas, SP. 2007

${ }^{4}$ GARCIA, A.; BARBAS, C. Gas Chromatography-Mass Spectrometry (GCMS)-Based Metabolomics. Metabolic Profilling: Methods and Protocols, Ed. Metz, T. O., Humana Press, Totowa, NJ, 2011.
} 